\title{
INFLUENCIA DE LA VISUALIZACIÓN EN TERAPIAS DE REHABILITACIÓN VIRTUAL ASISTIDAS POR ROBOTS
}

\author{
Luis D. Lledó, Santiago Ezquerro, Francisco J. Badesa, \\ Ramón Neco, José M. Sabater y Nicolás García-Aracil \\ Neuroingeneiría Biomédica, Universidad Miguel Hernández de Elche \\ \{llledo, sezquerro, fbadesa, ramon.neco, j.sabater, nicolas.garcia\}@umh.es
}

\section{Resumen}

La neurorehabilitación post-ictus basada en terapias virtuales se realiza junto con dispositivos visuales. El tipo de visualización puede influenciar en la calidad de la percepción de la tarea afectando al rendimiento sensomotor del paciente. El propósito de este trabajo es evaluar si existe diferencias en los patrones de movimientos cinemáticos cuando pacientes post-ictus realizan una tarea de alcance, visualizando un juego terapéutico virtual con dos diferentes tipos de representación del entorno virtual: 2D y 3D. En este estudio han participado nueve pacientes post-ictus recibiendo una terapia virtual asistida por el robot de rehabilitación PUPArm. Los pacientes debian realizar movimientos horizontales con el miembro superior para completar el objetivo principal de las tareas, el cuál consistía en alcanzar objetivos periféricos o perspectivos dependiendo del tipo de entorno virtual mostrado. Para realizar un análisis del rendimiento sensomotor de los pacientes se registran diferentes tipos de parámetros a partir de la adquisición de datos objetivos por parte del dispositivo robótico para evaluar la influencia de la visualización de la tarea.

Palabras clave: Realidad Virtual, Robótica de Rehabilitación, Post-ictus, Función Sensomotor, Extremidad Superior

\section{INTRODUCCIÓN}

La Realidad Virtual (RV) es una plataforma tecnológica que permite desarrollar entornos generados por computador donde los sujetos pueden explotar e interactuar con cualquier tipo de objetos o eventos para realizar tareas motores o de perspectiva. La principal característica que proporciona la RV es la posibilidad de repetir la misma tarea en cualquier momento, modificando factores como el nivel de complejidad, el tiempo y la intensidad de la práctica. De esta manera, la terapia virtual se puede utilizar para promover el aprendizaje y la rehabilitación motora debido a que la RV se puede ajustar para generar un entorno, escenario o actividad que permita al usuario practicar sus habil- idades motoras y mejorar la plasticidad neuronal dependiente de la experiencia [8]. La posibilidad de modificar factores como la repetición, la intensidad, el tiempo y la especificidad de las actividades de las terapias virtuales es beneficioso para este tipo de recuperación neuronal [10]. En los últimos años, algunos ensayos científicos y clínicos han demostrado la efectividad de la RV como una herramienta de intervención para la rehabilitación de diferentes lesiones con condiciones neurológicas especificas $[5,7]$.

em Varios estudios sugieren que la tecnología robótica se puede utilizar para mejorar la calidad y la evaluación en la evaluación en la rehabilitación neurológica [9], mejorando la productividad y reduciendo costes en este campo. Los recientes desarrollos en la tecnología robótica pueden ayudar a realizar una análisis más objetivo y fiable de las terapias que se aplican a los pacientes con lesiones neurológicas $[1,2]$. Esto se debe a la capacidad de registrar datos cinemáticos y cinéticos por parte de este tipo de dispositivos. A partir de estos datos se pueden extraer útiles marcadores para cuantificar el proceso de recuperación motor durante la terapia [20, 3, 17]. Recientemente, en [16] se ha demostrado que las sesiones de rehabilitación realizadas con dispositivos robóticos obtienen mejores resultados que las terapias convencionales durante la rehabilitación del miembro superior de pacientes post-ictus.

Con respecto a la rehabilitación virtual, se han desarrollado estudio fundamentados en sistemas RV con entornos implementados con gráficos $2 \mathrm{D}$ convencionales y otros con gráficos $3 \mathrm{D}$ [18, 14, 19]. Sin embargo, todavía no se han realizado realizado estudios sobre una comparación objetiva acerca de cómo afecta la visualización de gráficos $2 \mathrm{D}$ y entornos virtuales $3 \mathrm{D}$ a la percepción del movimiento en sujetos después del accidente cerebrovascular. Esto significa, que no existen evidencias que muestren si es mejor o no realizar tareas de rehabilitación virtual implementadas con gráficos $2 \mathrm{D}$ o $3 \mathrm{D}$. Utilizando gráficos $3 \mathrm{D}$ se puede aumentar la percepción visual de los elementos virtuales, de tal manera que las tareas basadas en actividades de la vida diaria son más similares 
a la realidad. Mientras que los gráficos $2 \mathrm{D}$ permiten una representación más simple de las tareas. Estas dos perspectivas se deben probar para evaluar qué tipo de representación visual proporciona una mejor calidad del rendimiento motor en términos de movimientos cinemáticos. Esta evaluación puede llevarse a cabo cuando el sujeto realiza el mismo movimiento para completar los objetivos utilizando ambos tipos de visualización. Por lo tanto, los dispositivos roboticos se pueden utilizar para restringir este movimiento y extraer objetivamente datos cuantitativos.

En este trabajo se evalúan los efectos de aplicar juegos terapéuticos con 2D o 3D en terapias virtuales asistidas por dispositivos robóticos y se analizan los resultados. De esta manera, se proporcionan datos cuantitativos para evaluar la influencia de la terapia virtual y valorar qué tipo de entorno virtual se ajusta mejor a cada paciente en términos de facilidad de uso, seguridad y comodidad. Por lo tanto, el objetivo principal de este estudio es determinar si existen diferencias en los parámetros cinemáticos de movimiento registrados por el dispositivo robótico que evalúan el rendimiento motor del paciente en tareas virtuales 2 D y 3D. Para ello, se ha diseñado dos tareas visuales modificando el nivel de inmersión utilizando gráficos bidimensionales y tridimensionales, pero se mantiene el objetivo cinemático dentro de las dos tareas visuales.

\section{MATERIALES Y MÉTODOS}

\subsection{Pacientes}

El estudio se ha realizado en un hospital de atención a pacientes crónicos y de larga estancia. El Comité de Ética Médica aprobó el protocolo experimental del estudio propuesto. El equipo médico ha sido el responsable de incluir pacientes que están recibiendo tratamiento de fisioterapia y terapia ocupacional. El personal médico ha informado debidamente a todos los pacientes y ellos dieron su consentimiento por escrito antes de iniciar el estudio, indicando que entendían el propósito y los requisitos del estudio. Los criterios de inclusión fueron: adultos con hemiparesia/hemiplejía secundaria con ictus en fase subaguda (entre 1 y 6 meses después de la lesión). Los criterios con respecto a las condiciones musculares de la extremidad superior fueron: i) tono muscular con puntuación por debajo de 2 en la Escala Modificada de Ashwoth [4], ii) balance equilibrio en la abducción del hombro y flexión del codo con base del Índice Motor $\geq 2$ [6]. Después del proceso de selección, han participado en el estudio nueve pacientes (edad 40-70 años) de ambos sexos.

\subsection{Sistema de neurorehabilitación}

El sistema de neurorrehabilitación utilizado para realizar la terapia motora y obtener toda la información objetiva sobre el estudio propuesto está formado por un sistema de robot PUPArm [2] y un subsistema de visualización. Este sistema fue diseñado y desarrollado por el Grupo de Neuroingeniería Biomédica en la Universidad Miguel Hernández de Elche como un robot de rehabilitación para pacientes con accidente cerebrovascular u otros trastornos neurológicos. El sistema de neurorrehabilitación se muestra en la Figura 1.

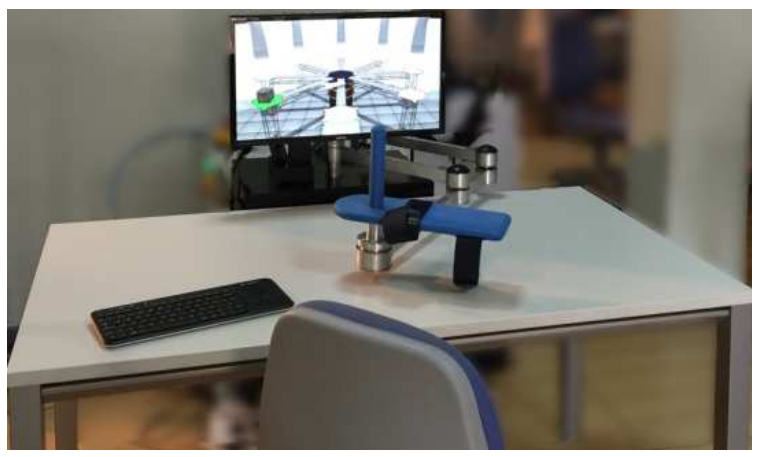

Figura 1: Sistema de neurorehabilitación basado en el robot PUPArm

El mecanismo robótico consiste en cuatro barras metálicas, similar al robot de rehabilitación MITMANUS [11]. Estas barras están conectados como un paralelogramo y se impulsan por módulos giratorios neumáticos. Esta estructura proporciona un manipulador planar con movimiento en dos dimensiones y dos grados de libertad. El manipulador permanece fijo en una mesa. Por consiguiente, el sistema sólo permite el movimiento horizontal de la extremidad superior de los sujetos, envolviendo la flexión y extensión del codo y el hombro, y la abducción y la aducción horizontal. Por otra parte, el subsistema de visualización se compone de un monitor de computador con un software desarrollado llamado REVIRE que se utiliza como sistema de simulación RV para visualizar actividades en coordinación con los movimientos del robot. Un ordenador se encarga de coordinar en tiempo real los actuadores neumáticos, los objetivos de las tareas y la información al usuario. El sistema es capaz de registrar información sobre el progreso del paciente en la rehabilitación, con base en parámetros como la posición, la velocidad y las fuerzas. Estos datos se procesan para ofrecer una evaluación objetiva al el terapeuta.

\subsection{Tareas virtuales}

La tarea virtual con el entorno $2 \mathrm{D}$ consiste en una ruleta formada por un objetivo central y ocho ob- 
jetivos periféricos. Estos objetivos son círculos con un radio de $1 \mathrm{~cm}$. Los ocho objetivos periféricos se distribuyen uniformemente en circunferencia alrededor del objetivo central a $10 \mathrm{~cm}$ de distancia. El objetivo principal de esta tarea es alcanzar uno de los ocho objetivos periféricos iniciando el movimiento desde el objetivo central, controlando del efector final del robot adjunto a la mano del sujeto. El correspondiente objetivo permanece iluminado. Para completar estos objetivos, la tarea se visualiza en el monitor del sistema de neurorrehabilitación con un refuerzo visual representado por un círculo blanco de $1 \mathrm{~cm}$ de radio para indicar la posición actual del efector final del robot. En la Figura 2 se muestra una captura de pantalla con el entorno 2D de la tarea virtual junto con información estructural.

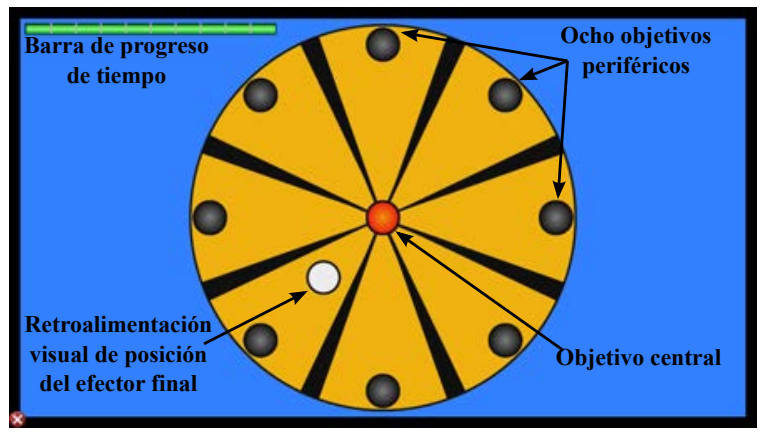

Figura 2: Escenario de la tarea 2D

Para completar este estudio comparativo, se ha diseñado una tarea virtual en 3D siguiendo los mismos criterios de objetivos utilizados en la tarea 2D, con el fin de realizar el mismo tipo de movimientos. En [12] se explica el patrón de implementación de simuladores virtuales utilizado para desarrollar esta tarea virtual 3D. El entorno simula una fábrica de cajas con una visión en perspectiva donde la escena converge al punto central de la pantalla. El escenario gráfico consta de ocho plataformas y un depósito central, los cuales representan a los ocho objetivos periféricos y al objetivo central de la tarea 2D. Las ocho plataformas también se colocan de manera uniforme alrededor del el depósito central. Para indicar al usuario la siguiente posición objetivo, se coloca una caja con comportamiento dinámico encima de una plataforma aleatoria. En este caso, el usuario controla el efector final del robot para gestionar una llave inglesa virtual con comportamiento cinemático, con el fin de recoger las cajas objetivo y soltarlas en el depósito central. La Figura 3 muestra una captura de pantalla con el entorno 3D de la tarea virtual junto con información estructural.

Básicamente, el propósito de esta tarea es la misma que la tarea 2D, pero con un nivel de visual-

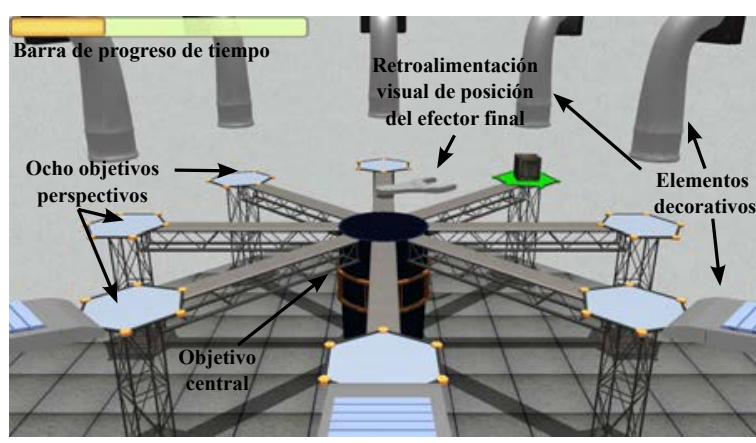

Figura 3: Escenario de la tarea 3D

ización diferente. El flujo de trabajo para cumplir la tarea virtual 3D es:

1. En primer lugar, el usuario debe aproximar la herramienta virtual al depósito central para iniciar la visualización de la posición del siguiente objetivo perspectivo.

2. Entonces, aparece aleatoriamente una caja en cualquiera de las ocho plataformas. La plataforma se ilumina como soporte visual, y la llave inglesa virtual se orienta de forma dinámica al objetivo posicional.

3. El usuario dispone de un tiempo limitado para recoger el caja objetivo. Este tiempo limitado se muestra en una barra de progreso colocado en el lateral superior izquierdo de la pantalla. Si no se alcanza el objetivo, la caja desaparece y se ejecuta el siguiente objetivo, apareciendo otra caja.

4. Cuando la llave virtual está cerca del objetivo, se captura la caja. A continuación, el usuario tiene que llevar la herramienta al depósito central para soltar la caja. También se ha incorporado un soporte sonoro para indicar que el objetivo se ha completado.

La funcionalidad y la estructura de ambas tareas virtuales son las mismas. Esto es necesario para una comparación objetiva de los valores de los parámetros obtenidos por el dispositivo robótico. En la Figura 4 se presenta una correlación estructural entre las tareas 2D y 3D. Este enfoque compara la misma situación con diferentes estímulos externos. El propósito de desarrollar un escenario en tridimensional es proporcionar una correlación más natural entre el movimiento del robot y la vista del usuario. En tareas 2D, cuando el usuario se acerca o se aleja el efector final con respecto del cuerpo, el elemento controlable en la tarea se mueve hacia arriba o hacia abajo de la pantalla, sin embargo, muchos pacientes tratan de forzar el efector final hacia arriba o hacia abajo para 
poner el elemento controlable en su lugar correspondiente en la pantalla. Por lo tanto, asociar el movimiento planar horizontal del dispositivo robótico con el movimiento vertical en la tarea $2 \mathrm{D}$ que aparece en pantalla, puede causar confusión en algunos pacientes. De esta manera, la tarea 3D replica en la pantalla el mismo tipo de movimiento del robot.
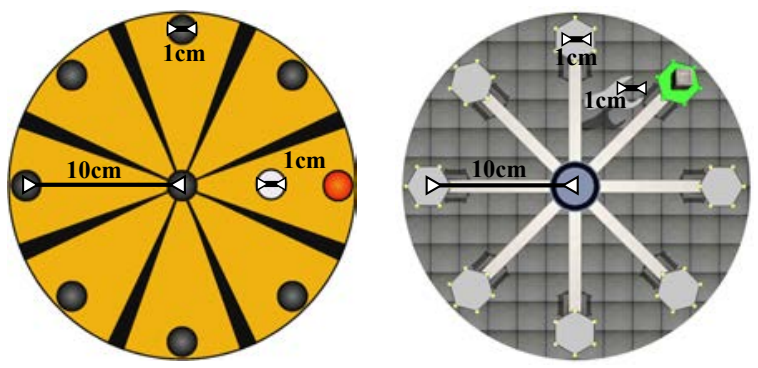

Figura 4: Correlación estructural entre las tareas 2D y $3 \mathrm{D}$ (vista cenital)

\subsection{Preparación y protocolo}

Durante un período de dos meses, el grupo de estudio ha recibido el tratamiento de la terapia asistida por el robot PUPArm con cuatro sesiones semanales de diez minutos, 36 sesiones en total. En la primera sesión, se lleva a cabo una evaluación general de la colocación del paciente para obtener parámetros como la altura de la pantalla o de la silla, y su rango de movilidad. Después, estos valores se utilizan durante las tareas. De esta manera, se consigue el rango funcional máximo del paciente específico. Antes de iniciar la sesión, el paciente se coloca en frente del dispositivo robótico en una posición cómoda con los parámetros obtenidos en la primera sesión de evaluación. El monitor encargado de ofrecer la retroalimentación visual se encuentra a $70 \mathrm{~cm}$ del paciente. Cada sesión se estructura en dos bloques de movimientos de entrenamiento, dependiendo de la tarea virtual. Entre cada bloque, los pacientes tenían períodos de descanso de tres minutos. El tiempo de la sesión se organiza de la siguiente manera

- En el primer bloque de movimientos, se elige al azar una de las dos tareas. A continuación, el paciente tiene que realizar 32 ensayos enfocándose en la tarea seleccionada aleatoriamente mediante movimientos globales, tanto de hombro y como de codo. Aproximadamente, este bloque se realiza en 4-5 minutos.

- Una vez que se han completado los 32 ensayos de la misma tarea, el paciente tiene un tiempo de descanso de tres minutos.
- Para finalizar la sesión, se realiza el segundo bloque de movimientos completando 32 ensayos de la otra tarea. Al igual que con el primer bloque, el paciente tiene que realizar los mismos movimientos globales y el tiempo necesario para realizar estos ensayos son aproximadamente 4-5 minutos también.

Los sujetos tenían que alcanzar uno de los ocho posibles objetivos y volver hacia el objetivo central para completar un ensayo. En la Figura 5 se muestra esquemáticamente el flujo de trabajo para realizar un ensayo. Para iniciar un ensayo, los sujetos tenían que mantener el elemento controlable a través de la efector final robótico durante 2 s encima del objetivo central. Entonces, uno de los objetivo alcanzables se ilumina para indicar la siguiente posición donde el paciente tenía que colocar el efector final. Para completar el movimiento, se da un tiempo limitado de 3s. Cuando el sujeto alcanza el objetivo, tiene que volver al objetivo central sin límite de tiempo. Por lo tanto, esta secuencia de movimientos se lleva a cabo en los 32 ensayos planteados, señalando objetivos de manera aleatoria. Este protocolo ha sido el mismo para las dos tareas virtuales, pero los elementos dentro de las tareas eran diferentes. A partir de los datos registrados por el dispositivo robótico se han obtenido los siguientes parámetros: 1) La velocidad máxima; 2) El tiempo de reacción; 3) La distancia total; 4) La distancia inicial; 5) La razón de movimiento inicial; 6) El error inicial de ángulo en la dirección de movimiento; 7) El tiempo total de la tarea y 8) La tasa de éxito. Se puede encontrar una explicación completa de estos parámetros en [13].

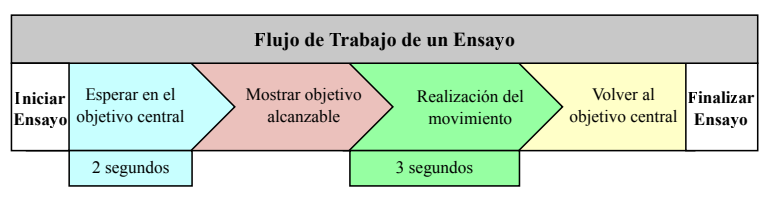

Figura 5: Flujo de trabajo completo de un ensayo

\section{RESULTADOS}

Durante 36 sesiones de tratamiento de terapia virtual asistida por el robot PUPArm se han adquirido datos objetivos y la Tabla 1 recoge las principales estadísticas descriptivas de cada paciente. Los resultados se presentan en una tabla de diez columnas para mostrar los valores de los parámetros cinemáticos que evalúan la calidad del funcionamiento motor del paciente. Cada parámetro contiene dos valores posibles, dependiendo del nivel de visualización de la tarea proporcionada al paciente. En este caso, se extrae de 
la tarea $2 \mathrm{D}$ y el otro de la tarea $3 \mathrm{D}$ para poder comparar los resultados numéricos. En la última columna se muestra la tasa de éxito a la hora de completar el objetivo alcanzable. La alta tasa de éxito entre el $95,10 \%$ y el $100 \%$ indica que el sistema es fácil de utilizar sin complicaciones para realizar las tareas virtuales. A simple vista no existen diferencias significativas en la tasa de éxito entre los dos tipos de visualización.

Con ambos valores extraídos de las tareas en 2D y $3 \mathrm{D}$, se ha realizado un análisis comparativo de estos parámetros a partir del cálculo de la variación en el rendimiento del paciente de los parámetros $3 \mathrm{D}$ con respecto a los parámetros $2 \mathrm{D}$. Se ha utilizado la Ecuación 1 para calcular los porcentajes de esta variación. Estos valores se reúnen en la Tabla 2 incluyendo la media, la desviación estándar, la mediana y el valor máximo-mínimo de cada parámetro para todos los datos. Los valores positivos indican el incremento de porcentaje, donde el parámetro extraído de la tarea en 3D es mayor que el mismo parámetro en la tarea 2D.

$$
\left(\frac{\text { dato } 3 D-\text { dato } 2 D}{\text { dato } 2 D}\right) * 100
$$

Estos parámetros se representan como diagramas de caja en la Figura 6 para proporcionar una visión general de la distribución de los datos. En un diagrama de cajas, las cajas están divididas por un segmento horizontal que indica la posición del valor de la mediana. Por lo tanto, se puede observar la relación entre este valor y los percentiles 25 th y 75 th, representada por la parte inferior y la parte superior de la caja. Las cajas se encuentran en un segmento cuyos extremos indican los valores mínimo y máximo del parámetro. En este diagrama de cajas, se han marcado los valores atípicos, por consiguiente aparece una distribución asimétrica en las cajas.

Para evaluar la función sensomotora después de la terapia virtual basado en la neurorrehabilitación asistida por robot, se comparan las trayectorias de movimiento realizadas por los sujetos en la primera y la última sesión. En ambas tareas, los pacientes efectuaron trayectorias más erráticas en la primera sesión cuando el sujeto intentaba llegar a los objetivos alcanzables. Sin embargo, los pacientes realizaron trayectorias más correctas cuando utilizaron la tarea con el entorno 2D. Las trayectorias presentan unas trayectorias más irregulares en la tarea 3D. Por otra parte, se llevaron a cabo menos desviaciones de trayectoria cuando los pacientes tenían que alcanzar el objetivo central en la tarea 2D. Con respecto a la primera sesión, en la tarea 3D las trayectorias entre el objetivo alcanzable y el objetivo central presentan una mayor longitud que en la tarea 2D y el error en la desviación y el tiempo para alcanzar los objetivos es más alta (véase la Tabla 2). Por lo tanto, los pacientes presentan más dificultades a la hora de alcanzar los objetivos de la tarea 3D cuando comenzaron la terapia, utilizando el sistema propuesto. En la última sesión, las trayectorias mejoraron considerablemente al reducir el error en la desviación.

\section{DISCUSIONES}

En este capítulo se ha presentado un estudio sobre la influencia de la aplicación de juegos terapéuticos 2D o 3D en la realización de la rehabilitación del miembro superior en pacientes después del accidente cerebrovascular. En la actualidad, este tipo de estudios no se han abordado todavía en la literatura científica o los temas tratados en este campo son discutidos por evaluaciones subjetivas. Por estas razones, se ha evaluado de forma objetiva una correlación cuantitativa entre la función motora de las extremidades superiores y la visualización de la tarea de alcance a través del cálculo de parámetros cinemáticos proporcionadas por el dispositivo robótico llamado PUPArm. Los resultados fueron similares en la mayoría de los parámetros, pero se han encontrado algunas pequeñas diferencias en el rendimiento sensomotor dependiendo de la visualización de la tarea basada en objetivos periféricos o perspectivos. Cada paciente ha logrado resultados similares cuando realizó las tareas con ambos entornos durante todas las sesiones de la terapia. Sin embargo, algunos pacientes obtienen mejores resultados que otros, mostrando una variación en las capacidades sensomotoras. Estos cambios pueden deberse a la edad, daños motores o el nivel de lesión cerebral que afecta a la eficiencia de los procesos cognitivos y fisiológicos.

Uno de los propósitos mas importantes de las tareas era realizar trayectorias rectas para alcanzar los objetivos. En las primeras sesiones, la mayoría de los objetivos en ambas tareas se alcanzaban con trayectorias erráticas. Para los mismos objetivos periféricos y perspectivos, las trayectorias presentan diferentes desviaciones en cada ensayo. Este hecho sucedió para todos los pacientes. Sin embargo, los objetivos alcanzables que necesitaban trayectorias diagonales se lograron con mejores movimientos cinemáticos en la tarea 2D, llevando a cabo trayectorias más rectilíneas. En las tareas 3D, se observan trayectorias desviadas en prácticamente todos los objetivos. En lo que respecta al objetivo central, las trayectorias fueron más precisas y con menos desviación en la tarea 2D. El mejor rendimiento sensomotor en las tar- 
Tabla 1: Datos adquiridos por el dispositivo robótico.

\begin{tabular}{|c|c|c|c|c|c|c|c|c|c|}
\hline Paciente & Tarea & $\begin{array}{c}\text { Velocidad } \\
\text { Máxima }(\mathrm{mm} / \mathrm{s})\end{array}$ & $\begin{array}{c}\text { Tiempo de } \\
\text { Reacción(s) }\end{array}$ & $\begin{array}{c}\text { Distancia } \\
\text { Total }(\mathrm{mm})\end{array}$ & $\begin{array}{c}\text { Distancia } \\
\text { Inicial }(\mathrm{mm})\end{array}$ & $\begin{array}{c}\text { Razón de } \\
\text { Mov. Inicial }\end{array}$ & $\begin{array}{c}\text { Error Inicial } \\
\text { Ángulo }\left(^{\circ}\right)\end{array}$ & Tiempo & $\begin{array}{l}\text { Tasa de } \\
\text { Éxito(\%) }\end{array}$ \\
\hline \multirow[t]{2}{*}{1} & $2 \mathrm{D}$ & 104.13 & 0.69 & 104.27 & 72.45 & 0.70 & 1.11 & 7.11 & 99.61 \\
\hline & $3 \mathrm{D}$ & 115.56 & 0.89 & 114.41 & 83.01 & 0.74 & 1.13 & 8.18 & 99.61 \\
\hline \multirow[t]{2}{*}{2} & $2 \mathrm{D}$ & 57.07 & 0.65 & 114.55 & 41.60 & 0.38 & 3.21 & 10.35 & 100 \\
\hline & $3 \mathrm{D}$ & 58.64 & 0.71 & 121.53 & 42.01 & 0.36 & 3.17 & 11.38 & 98.64 \\
\hline \multirow[t]{2}{*}{3} & $2 \mathrm{D}$ & 91.81 & 0.85 & 116.94 & 58.38 & 0.52 & 2.05 & 11.81 & 100 \\
\hline & $3 \mathrm{D}$ & 92.58 & 1.09 & 119.06 & 60.60 & 0.53 & 1.87 & 13.59 & 100 \\
\hline \multirow[t]{2}{*}{4} & $2 \mathrm{D}$ & 118.30 & 0.71 & 123.82 & 69.13 & 0.61 & 1.57 & 13.45 & 99.67 \\
\hline & $3 \mathrm{D}$ & 134.66 & 0.89 & 149.68 & 78.62 & 0.60 & 1.67 & 16.26 & 98.58 \\
\hline \multirow[t]{2}{*}{5} & $2 \mathrm{D}$ & 153.19 & 0.88 & 197.90 & 95.59 & 0.61 & 1.71 & 15.41 & 98.83 \\
\hline & $3 \mathrm{D}$ & 153.40 & 1.04 & 250.27 & 97.45 & 0.51 & 2.53 & 27.04 & 95.10 \\
\hline \multirow[t]{2}{*}{6} & $2 \mathrm{D}$ & 45.94 & 0.40 & 110.07 & 33.96 & 0.32 & 3.56 & 12.13 & 98.83 \\
\hline & $3 \mathrm{D}$ & 46.57 & 0.48 & 111.14 & 32.89 & 0.31 & 3.77 & 13.48 & 97.01 \\
\hline \multirow[t]{2}{*}{7} & $2 \mathrm{D}$ & 63.24 & 0.64 & 120.42 & 42.98 & 0.37 & 3.19 & 16.07 & 97.13 \\
\hline & $3 \mathrm{D}$ & 60.49 & 0.77 & 121.61 & 41.68 & 0.36 & 3.17 & 15.99 & 95.53 \\
\hline \multirow[t]{2}{*}{8} & $2 \mathrm{D}$ & 110.09 & 0.52 & 105.96 & 69.78 & 0.68 & 1.34 & 8.03 & 98.96 \\
\hline & $3 \mathrm{D}$ & 113.72 & 0.71 & 115.34 & 74.28 & 0.67 & 1.37 & 11.76 & 98.96 \\
\hline \multirow[t]{2}{*}{9} & $2 \mathrm{D}$ & 112.37 & 0.73 & 130.40 & 75.48 & 0.64 & 1.73 & 12.36 & 100 \\
\hline & $3 \mathrm{D}$ & 121.37 & 1.05 & 157.78 & 83.21 & 0.61 & 1.88 & 13.33 & 98.27 \\
\hline
\end{tabular}

Tabla 2: Variación de los parámetros 3D con respecto a los parámetros 2D [\%].

\begin{tabular}{|ccccccccc|}
\hline Paciente & $\begin{array}{c}\text { Velocidad } \\
\text { Máxima }\end{array}$ & $\begin{array}{c}\text { Tiempo de } \\
\text { Reacción }\end{array}$ & $\begin{array}{c}\text { Distancia } \\
\text { Total }\end{array}$ & $\begin{array}{c}\text { Distancia } \\
\text { Inicial }\end{array}$ & $\begin{array}{c}\text { Razón de } \\
\text { Mov. Inicial }\end{array}$ & $\begin{array}{c}\text { Error Inicial } \\
\text { Ángulo }\end{array}$ & $\begin{array}{c}\text { Tiempo } \\
\text { Tasa de } \\
\text { Éxito }\end{array}$ \\
\hline 1 & 10.97 & 29.27 & 9.73 & 14.57 & 5.79 & 1.55 & 15.13 & 0 \\
2 & 2.75 & 9.35 & 6.09 & 0.99 & -4.69 & -2.14 & 10 & -1.36 \\
3 & 0.83 & 27.80 & 1.80 & 3.80 & 2.14 & -8.99 & 15.12 & 0 \\
4 & 13.83 & 25.26 & 20.89 & 13.72 & -3.07 & 6.74 & 20.89 & -1.09 \\
5 & 0.13 & 18.92 & 26.46 & 1.94 & -17.28 & 47.90 & 75.47 & -3.78 \\
6 & 1.36 & 20.11 & 0.97 & -3.09 & -3.79 & 5.99 & 11.12 & -1.84 \\
7 & -4.35 & 10.99 & 0.98 & -3.03 & -3.06 & -0.49 & -0.47 & -1.64 \\
8 & 3.29 & 37.77 & 8.85 & 6.45 & -1.16 & 2.18 & 46.58 & 0 \\
9 & 8 & 42.93 & 20.99 & 10.24 & -4.77 & 8.99 & 7.83 & -1.73 \\
\hline MEDIA & 4.09 & 24.71 & 10.75 & 5.07 & -3.32 & 6.86 & 22.41 & -1.27 \\
STD & 5.42 & 10.60 & 9.14 & 6.27 & 5.93 & 15.37 & 22.42 & 1.21 \\
MEDIANA & 2.75 & 25.26 & 8.85 & 3.80 & -3.07 & 2.18 & 15.12 & -1.36 \\
MAX & 13.83 & 42.93 & 26.46 & 14.57 & 5.79 & 47.90 & 75.47 & 0 \\
MIN & -4.35 & 9.35 & 0.97 & -3.09 & -17.28 & -8.99 & -0.47 & -3.78 \\
\hline
\end{tabular}

eas 2D puede sugerir que los entornos $2 \mathrm{D}$ son más factibles, evidentes y cómodos cuando los pacientes empiezan a utilizar el sistema, por estas razones: las trayectorias eran más rectilíneas y tenían menos desviaciones. En la última sesión, las trayectorias de movimiento se corrigieron significativamente hasta el punto de lograr caminos casi sin desviación, mientras que los objetivos se alcanzan satisfactoriamente con trayectorias estables. Comparando de los resultados entre la primera y la última sesión se puede observar que las trayectorias mejoran con la experiencia del paciente. En general, las tasas de éxito en todas las sesiones durante ambas tareas eran bastante altas. Los objetivos se han alcanzado prácticamente en todos los ensayos por parte de todos los pacientes. Los altos valores de la tasa de éxito insinúan que las tareas no eran complejos y los objetivos fueron reconocidos con claridad.

Por otra parte, las diferencias entre las tareas en términos de parámetros cinemáticos y cinéticos se pueden encontrar en la Tabla 1, la Tabla 2 y la Figura 6. Para todos los pacientes, el tiempo de reacción en la tarea $3 \mathrm{D}$ fue superior que en la tarea 2D, implicando que el aumento del nivel de inmersión en el entorno provoca distracciones innecesarias a los pacientes. Así, el nivel de concentración del paciente aumenta con menos nivel de detalle en el entorno virtual. Durante la realización de las trayectorias para alcanzar los objetivos, el manipulador del robot se desplaza en menor medida en la tarea 2D. Por lo tanto, la distancia total ha sido mayor en la tarea 3D para todos los pacientes. En consecuencia, el tiempo total para completar todos los ensayos fue superior en la tarea 3D. Sólo un paciente ha necesitado menos tiempo para realizar la tarea 3D.

Puede ser que los pacientes guían mejor el manipulador robótico cuando están observando un entorno 2D. En la tarea 3D, la profundidad del escenario aumenta el nivel de dificultad para completar los objetivos. Los pacientes se han adaptado 


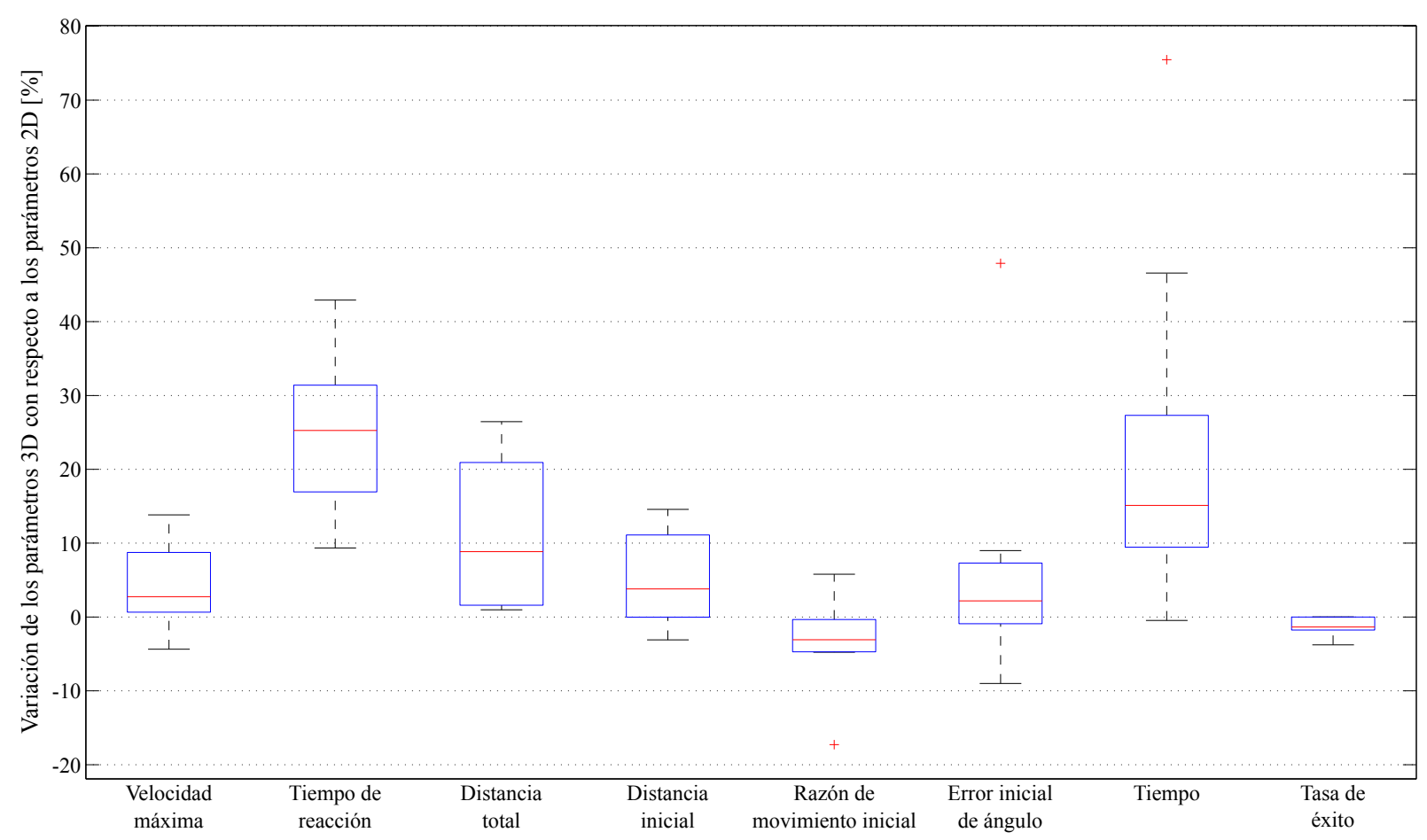

Figura 6: Análisis estadístico de los datos adquiridos, representado en un diagrama de cajas

mejor a la tarea 2D. Aunque la distancia total fue mayor en la tarea $3 \mathrm{D}$, los tiempos para completar ambas tareas no difieren sustancialmente debido a los pacientes lograron una velocidad máxima de movimiento en la tarea 3D. Esto significa que se produce la realización de movimiento de un manera más natural y dinámica. Todos los ensayos se pueden realizar de una manera óptima siguiendo una trayectoria recta desde el objetivo central y el objetivo alcanzable, y viceversa. Sin embargo, se produce una desviación cuando los pacientes comenzaban el movimiento para completar todos los ensayos. Esta desviación implica que los pacientes realizan una ruta incorrecta antes de que la dirección de trayectoria se corrigiera para alcanzar la ruta óptima. Esta situación se acentúa más en la tarea 3D en casi todos los pacientes, como se observa a través del análisis de los parámetros: distancia inicial, la razón de movimiento inicial y el error inicial del ángulo en la dirección del movimiento.

En conclusión, el objetivo principal de este estudio era verificar si habían diferencias en los patrones de los movimientos cinemáticos de los pacientes con ictus asistidos por un dispositivo robótico para situaciones donde se visualizaban entornos dos y tres dimensiones. A pesar de la similitud en los resultados, la hipótesis que consiste en mostrar un entorno de visualización más natural aumentando el nivel de inmersión, no ha proporcionado muchas mejoras con respecto a un entorno más simple. Por lo tanto, el uso de entornos 2D en la terapia virtual puede ser una manera más factible, apropiada y cómoda para llevar a cabo tareas de rehabilitación de las extremidades superiores de pacientes después del accidente cerebrovascular. El conocer qué entorno virtual es más apropiado para cada usuario permite implementar terapias con mejores instrumentos de evaluación que pueden ser adaptadas a las necesidades y a las limitaciones del paciente [15]. Esto proporciona muchos beneficios en el entorno clínico para mejorar el curso de la rehabilitación de la función sensomotora y reducir los tiempos de recuperación.

\section{Agradecimientos}

Este trabajo ha sido financiado por la Comisión Europea a través del proyecto HomeRehab: Echord++ (GA 601116) y por el Ministerio de Economía y Competitividad a través del proyecto DPI2015-70415-C2-2-R. Estamos muy agradecidos al Hospital de la Pedrera por su colaboración en este estudio.

\section{Referencias}

[1] Badesa, F. J., Morales, R., Garcia-Aracil, N., Sabater, J. M., Perez-Vidal, C., y Fernandez, E., (2012), "Multimodal interfaces to improve therapeutic outcomes in robotassisted rehabilitation," Systems, Man, and Cybernetics, Part C: Applications and Reviews, IEEE Transactions on, vol. 42, no. 6, pp. $1152-1158$. 
[2] Badesa, F. J., Llinares, A., Morales, R., Garcia-Aracil, N., Sabater, J. M., y PerezVidal, C., (2014), "Pneumatic planar rehabilitation robot for post-stroke patients," Biomedical Engineering: Applications, Basis and Communications, vol. 26, no. 02.

[3] Bertomeu-Motos, A., Lledó, L. D., Díez, J. A., Catalan, J. M., Ezquerro, S., Badesa, F. J., y Garcia-Aracil, N., (2015), "Estimation of human arm joints using two wireless sensors in robotic rehabilitation tasks," Sensors, vol. 15, no. 12, pp. 30571-30583.

[4] Bohannon, R. W. y Smith, M. B., (1987), "Interrater reliability of a modified ashworth scale of muscle spasticity," Physical therapy, vol. 67 , no. 2, pp. 206-207.

[5] Burdea, G., "Keynote address: Virtual rehabilitation-benefits and challenges," in 1st International Workshop on Virtual Reality Rehabilitation (Mental Health, Neurological, Physical, Vocational) VRMHR. sn, (2002), pp. $1-11$.

[6] Collin, C. y Wade, D., (1990), "Assessing motor impairment after stroke: a pilot reliability study." Journal of Neurology, Neurosurgery \& Psychiatry, vol. 53, no. 7, pp. 576-579.

[7] Crosbie, J., Lennon, S., Basford, J., y McDonough, S., (2007), "Virtual reality in stroke rehabilitation: still more virtual than real," Disability and rehabilitation, vol. 29, no. 14, pp. 1139-1146.

[8] Doyon, J. y Benali, H., (2005), "Reorganization and plasticity in the adult brain during learning of motor skills," Current opinion in neurobiology, vol. 15, no. 2, pp. 161-167.

[9] Garcia, N., Sabater-Navarro, J. M., Gugliemeli, E., y Casals, A., (2011), "Trends in rehabilitation robotics," Medical and Biological Engineering and Computing, vol. 49, no. 10, pp. 1089-1091.

[10] Kleim, J. A. y Jones, T. A., (2008), "Principles of experience-dependent neural plasticity: implications for rehabilitation after brain damage," Journal of speech, language, and hearing research, vol. 51, no. 1, pp. S225S239.

[11] Krebs, H. I., Hogan, N., Aisen, M. L., y Volpe, B. T., (1998), "Robot-aided neurorehabilitation," Rehabilitation Engineering, IEEE Transactions on, vol. 6, no. 1, pp. 75-87.
[12] Lledó, L. D., Badesa, F. J., Almonacid, M., Cano-Izquierdo, J. M., Sabater-Navarro, J. M., Fernández, E., y Garcia-Aracil, N., (2015), "Supervised and dynamic neuro-fuzzy systems to classify physiological responses in robot-assisted neurorehabilitation," PloS one, vol. 10, no. 5, p. e0127777.

[13] LLinares, A., Badesa, F. J., Morales, R., Garcia-Aracil, N., Sabater, J., y Fernandez, E., (2013), "Robotic assessment of the influence of age on upper-limb sensorimotor function," Clinical interventions in aging, vol. 8, p. 879 .

[14] Merians, A. S., Poizner, H., Boian, R., Burdea, G., y Adamovich, S., (2006), "Sensorimotor training in a virtual reality environment: does it improve functional recovery poststroke?" Neurorehabilitation and neural repair, vol. 20, no. 2, pp. 252-267.

[15] Morales, R., Badesa, F. J., Garcia-Aracil, N., Perez-Vidal, C., Sabater, J. M., Papaleo, E., Salerno, A., Zollo, L., y Guglielmelli, E., (2014), "Patient-tailored assistance," Robotics and Automation Magazine.

[16] Norouzi-Gheidari, N., Archambault, P. S., y Fung, J., (2012), "Effects of robot-assisted therapy on stroke rehabilitation in upper limbs: systematic review and meta-analysis of the literature," Journal of rehabilitation research and development, vol. 49, no. 4, p. 479.

[17] Papaleo, E., Zollo, L., Garcia-Aracil, N., Badesa, F., Morales, R., Mazzoleni, S., Sterzi, S., y Guglielmelli, E., (2015), "Upperlimb kinematic reconstruction during stroke robot-aided therapy," Medical $\&$ biological engineering $\&$ computing, vol. 53, no. 9, pp. 815-828.

[18] Rose, F. D., Brooks, B. M., y Rizzo, A. A., (2005), "Virtual reality in brain damage rehabilitation: review," CyberPsychology $\& B$ Behavior, vol. 8, no. 3, pp. 241-262.

[19] Saposnik, G., "Virtual reality in stroke rehabilitation," in Ischemic Stroke Therapeutics. Springer, (2016), pp. 225-233.

[20] Volpe, B. T., Huerta, P. T., Zipse, J. L., Rykman, A., Edwards, D., Dipietro, L., Hogan, N., y Krebs, H. I., (2009), "Robotic devices as therapeutic and diagnostic tools for stroke recovery," Archives of neurology, vol. 66, no. 9, pp. 1086-1090. 